\title{
Electrostatically Shielded Quantum Confined Stark Effect Inside Polar Nanostructures
}

\author{
Spilios Riyopoulos
}

Received: 5 December 2008/Accepted: 12 May 2009/Published online: 30 May 2009

(C) to the authors 2009

\begin{abstract}
The effect of electrostatic shielding of the polarization fields in nanostructures at high carrier densities is studied. A simplified analytical model, employing screened, exponentially decaying polarization potentials, localized at the edges of a QW, is introduced for the ESshielded quantum confined Stark effect (QCSE). Wave function trapping within the Debye-length edge-potential causes blue shifting of energy levels and gradual elimination of the QCSE red-shifting with increasing carrier density. The increase in the $e-h$ wave function overlap and the decrease of the radiative emission time are, however, delayed until the "edge-localization" energy exceeds the peak-voltage of the charged layer. Then the wave function center shifts to the middle of the $\mathrm{QW}$, and behavior becomes similar to that of an unbiased square QW. Our theoretical estimates of the radiative emission time show a complete elimination of the QCSE at doping densities $\geq 10^{20} \mathrm{~cm}^{-3}$, in quantitative agreement with experimental measurements.
\end{abstract}

\section{Introduction}

The presence of a strong, inherent polar electric field in GaN [1] causes the well-known quantum confined Stark effect [2-4] (QCSE) regarding carrier behavior inside a QW (Fig. 1a). The separation of the center of charge between electron and hole wave functions, caused by the polar $E$-field, reduces mutual overlap and the related

S. Riyopoulos $(\square)$

Science Applications International Corporation, McLean, VA 22102, USA

e-mail: spilios.riyopoulos@ saic.com emission probability. The lowering of the confined energy levels, relative to the unperturbed square QW, causes redshifting of the emitted radiation during electron-hole recombination. This effect has been the subject of extensive perturbative [5] as well as non-perturbative analytic treatments [6-9], including excitonic effects [10-14]. In general earlier analytic theories neglected the modifications to the (intrinsic polar or externally applied) $E$-field caused by the charge separation and the resulting dielectric shielding, assuming in effect very low carrier densities.

At high carrier densities, charge separation and dipole field formation is sufficient to cause shielding of the intrinsic polarization $E$-field [15]. The resulting potential gradient across the QW is not uniform, and most of the potential drop is localized across charged layers formed at the edges of the QW (Fig. 1b). The electric gradient scale is of the order of the Debye length. For densities near $10^{19} \mathrm{~cm}^{-3}$ the Debye length shrinks down to $\mathrm{nm}$-scale (Fig. 1c), and the potential drop is mostly localized at the QW edges while the QW interior is nearly fieldfree (shielding of the intrinsic $E$-field). This constitutes the ES-shielded QCSE. It has been anticipated [16] that the shielding of the interior $E$-field would reduce or even eliminate the QCSE at densities $10^{19} \mathrm{~cm}^{-3}$. Detailed numerical simulations, employing the self-consistent Poisson-Schrodinger equations [17] have showed that a much higher than expected carrier density, near $10^{20} \mathrm{~cm}^{-3}$, is required to eliminate the QCSE for QWs wider than $5 \mathrm{~nm}$. This has been attributed to the persistence of carrier confinement in the potential dips at the QW edges, even when the electric field is screened out from the middle. However, an analytic treatment examining the carrier behavior in the ES-shielded QCSE is so far lacking.

This study focuses in finding solutions for the confined carrier wave functions by solving the one-particle 


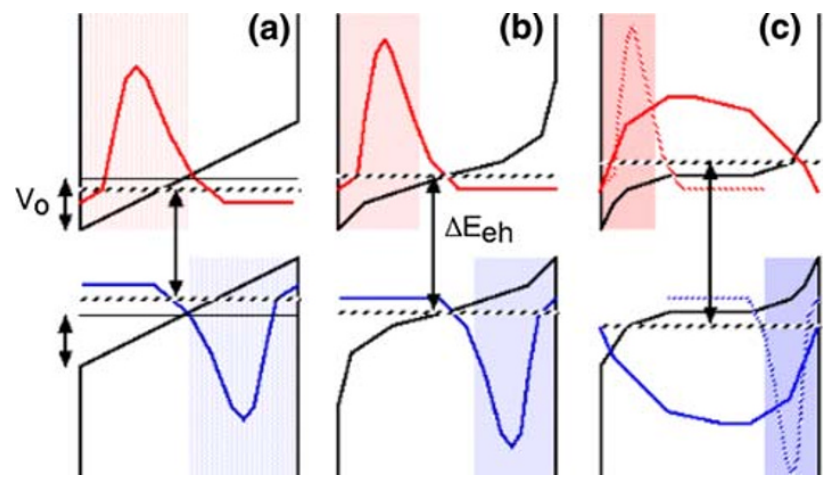

Fig. 1 a Internal polarization field causes separation in the carrier wave function centers and charge separation. b As carrier density increases the electric field is shielded (reduced) at the center of the well and most of the potential drop occurs near the edges. Wave fucntions are localized at the edges. The energy level separation increases (blue shifts) with increasing wave function confinement (constriction). c At even higher densities the electric field is completely shielded at the center and the voltage drop is localized at nanometer-width charged layers (plasma sheaths). Eventually the energy level is pushed above the edge-well depth $V_{\mathrm{o}}$ and the wave function expands to occupy the entire QW width, for a complete "rectification" of the QCSE

Schrodingers' equation. To gain insight the following simplifying assumptions are used: (a) The shielded potential has exponentially decaying profile on the Debye length $\sim \lambda_{\mathrm{D}}$ scale; (b) the peak-to-peak shielded voltage is a given function of the carrier density and the intrinsic polarization strength $\mathcal{E}_{\mathrm{o}}$; and (c) excitonic effects are ignored.

The shielded potential results from a self-consistent solution of Poisson's equation for point-like charges obeying Fermi statistics [15]. Neglecting the charge spreading of the carrier wave function is not too severe when the carrier localization length $\sim \lambda_{\mathrm{D}}$ is much smaller than the QW width $L$. When the Fermi level separation from the lowest occupied levels is much larger than $\kappa T$, i.e., for nearly Maxwellian distributions, the shielded potential is well approximated by a symmetric profile $V_{\mathrm{sh}}(x)=$ $V_{\mathrm{o}} \sin h\left(\kappa_{\mathrm{D}} x\right) / \sin h\left(\kappa_{\mathrm{D}} L / 2\right)$. The exponentially decaying profiles remain a reasonable approximation for FermiDirac distributions in general.

We obtain results based on: (a) a second order perturbative expansion; (b) non-perturbative series expansion; and (c) a numerical solution of Scrodinger's equation for the carrier envelope wave function. The analytic expressions for the energy levels from (a) are evaluated against numerical the results from (c). The infinite $\lambda_{D}$, zero shielding limit reverts to the original (unshielded) QCSE results.

Our analytic models find that increasing the carrier density causes an increase (blue shifting) of the energy levels relative to the unshielded (red-shifted) QCSE values. The confined energy levels asymptote to the values for a flat square QW, and the red shift is effectively eliminated, for densities $\geq 10^{19} \mathrm{~cm}^{-3}$. The perturbative energy levels agree with the numerical values at low $V_{\mathrm{p}}$, and become inaccurate when the polarization voltage $e V_{\mathrm{p}}=e \mathcal{E}_{\mathrm{o}} L$ exceeds the energy of the fundamental confined mode in a square QW. Numerical solutions of the Schrodinger equation for high polarization, relevant to GaN parameters, show that at high $V_{\mathrm{p}}$ the perturbation results overestimate the energy level shifts by a factor of 2 , but they provide the correct trends over the entire range.

The dependence of the characteristic emission time on the carrier density is computed based on the numerically evaluated eigenfunctions. Despite the adopted simplifications these results reproduce the three order of magnitude increase in the emission rate between densities $10^{19}$ and $10^{21}$, leading to a complete rectification of the QCSE, as was reported from experimental and detailed computations in Ref. [17].

Interestingly, it is found that elimination of the QCSErelated energy red-shift clearly precedes the recovery of the radiative emission time: the energy red-shifting is gradually eliminated between densities $10^{17} \mathrm{~cm}^{-3}$ and $10^{19} \mathrm{~cm}^{-3}$ while the emission probability is restored at higher densities between $10^{19} \mathrm{~cm}^{-3}$ and $10^{20} \mathrm{~cm}^{-3}$. The first result agrees with the energy recovery behavior obtained in [16] while the emission probability behavior agrees with the results in [17]. The delay in the restoration of the emission probability is explained in terms of carrier trapping at the QW edge.

\section{QW Eigen Modes with ES-shielded Polar Potential}

We investigate the wave function profiles and the structure of the energy spectrum inside QWs in the presence of an ES-shielded polarization potential. It can be shown (Appendix 1) that the self-consistent charged layer (plasma sheath) potentials can be reasonably approximated by exponentially decaying

$\Phi_{\mathrm{p}}(x)=-V_{\mathrm{o}} \frac{\exp \left[-\kappa_{\mathrm{D}} x\right]}{\exp \left[-\kappa_{\mathrm{D}} L / 2\right]}$

where $\kappa_{\mathrm{D}}=a / \lambda_{\mathrm{D}}$ scales as the inverse Debye length and $a$ is of order unity. The peak amplitude $V_{\mathrm{o}}$ here is taken equal to half the intrinsic "polarization voltage" $V_{\mathrm{p}} \equiv \mathcal{E}_{\mathrm{o}} L$. The value $\Phi_{\mathrm{p}}(0)=0$ at mid-point equals the bottom energy for a polarization-free square well (Fig. 2), and serves as the reference point for electron energy levels. Hole levels are measured from the bottom of the valence well. The above symmetric potential applies for low carrier density and a Fermi level near the mid bandgap. For high doping the reference point $x_{\mathrm{o}}$ defined by $\Phi_{\mathrm{p}}\left(x_{\mathrm{o}}\right)=0$ moves closer 

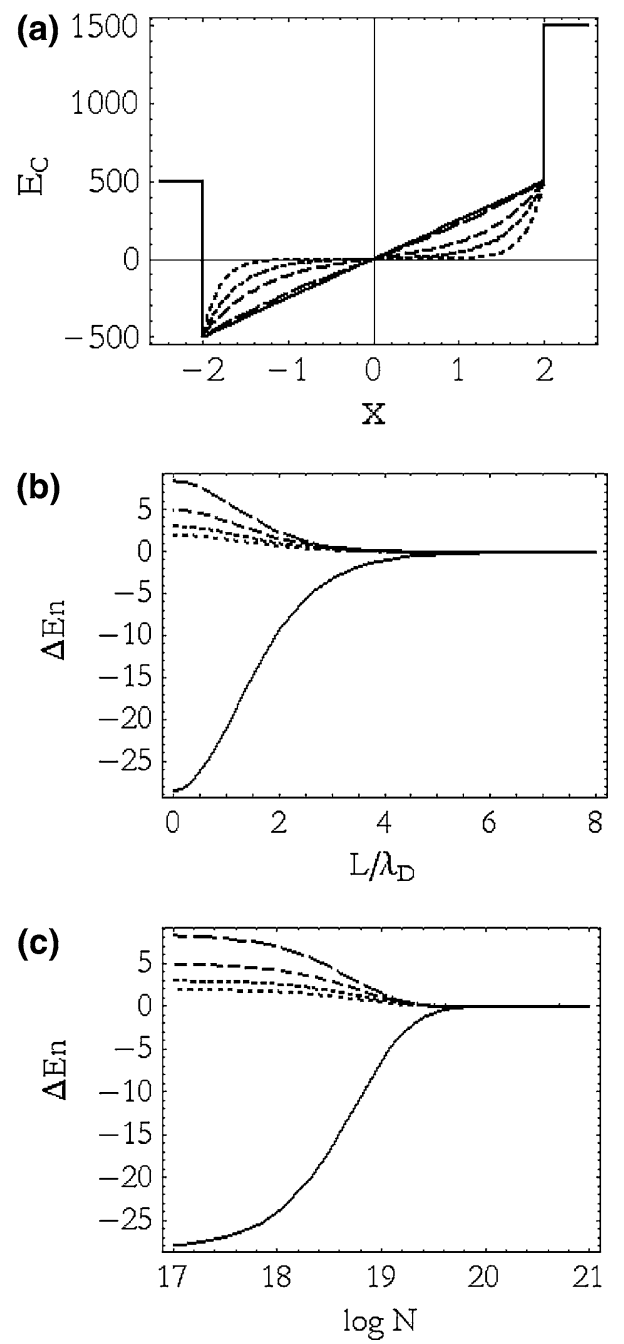

Fig. 2 a Profile of a QW conduction band with a ES-shielded polarization field for characteristic shielding distance (Debye length) $\lambda_{\mathrm{D}}=8 L, L / 2, L / 6, L / 10, L / 20$, longer to shorter dash lines. b Energy correction (meV) versus $L / \lambda_{\mathrm{D}}$, for the lowest five QW modes with $V_{\mathrm{o}}=25 \mathrm{meV}$ and $\mathrm{QW}$ width $L=8 \mathrm{~nm}$. c Same versus carrier density $N$ corresponding to $\lambda_{\mathrm{D}}$

to the left (right), with unequal edge potentials $-V_{\mathrm{p}}(-L / 2)$ $>V_{\mathrm{p}}(L / 2)\left(-V_{\mathrm{p}}(-L / 2)>V_{\mathrm{p}}(L / 2)\right)$ for $N$-doped $(P$-doped $)$ materials. For analytic simplicity this study will retain the symmetric potential.

Expressing the slowly varying envelope wave function in separable coordinates as $\Psi_{n, k_{y}, k_{z}}(x, y, z)=\psi_{n}(x)$ $\exp \left[-\mathrm{i} k_{y} y\right] \exp \left[-\mathrm{i} k_{z} z\right]$ casts the 1-D Schrodinger's equation along $x$ as

$-\frac{\hbar^{2}}{2 m^{*}} \frac{d^{2}}{\mathrm{~d} x^{2}} \psi_{n}+e \Phi_{\mathrm{p}}(x) \psi_{n}=E_{n} \psi_{n}$

where $E_{n}=E_{n, k_{y}, k_{z}}-\hbar^{2} k_{y}^{2} / 2 m^{*}-\hbar^{2} k_{z}^{2} / 2 m^{*}$ is the net energy contribution from the motion across the well, and $k_{y}, k_{z}$ correspond to the continuous spectrum along the $\mathrm{QW}$.
Analytic solutions of (2) are obtained from second order perturbation theory, in terms of an expansion in unperturbed square well modes $\psi_{n}^{(0)}=\sqrt{2 / L} \sin [(n \pi / 2) x], \quad E_{n}^{(0)}=$ $n^{2} \hbar^{2} \pi^{2} / 2 m^{*} L^{2}$

$E_{n}=E_{n}^{(0)}+H^{\prime}{ }_{n n}+\sum_{l \neq n} \frac{\left|H_{n l}{ }^{\prime}\right|^{2}}{E_{n}^{(0)}-E_{l}^{(0)}}$

with

$H_{n l}{ }^{\prime}=\frac{V_{\mathrm{o}}}{\sinh \left(k_{\mathrm{D}} L / 2\right)} \frac{2}{L} \int_{-L / 2}^{L / 2} \mathrm{~d} x \sin h\left(\kappa_{\mathrm{D}} x\right) \sin \left[\frac{n \pi}{2} x\right] \sin \left[\frac{l \pi}{2} x\right]$

A change of variable $s=\frac{1}{L}\left(x+\frac{L}{2}\right)$ transforms the integral in the rhs of (4) into

$2 \int_{-1}^{1} \mathrm{~d} s \sin h\left(\kappa_{\mathrm{D}} L\left(s-\frac{1}{2}\right)\right) \sin [n \pi s] \sin [l \pi s]$
$=2\left(\kappa_{\mathrm{D}} L\right) \frac{2 n l \pi^{2}\left(1-(-1)^{n+l}\right)^{2}}{\left[\left(n^{2} \pi^{2}+l^{2} \pi^{2}+\kappa_{\mathrm{D}}^{2} L^{2}\right)^{2}-4 n^{2} l^{2} \pi^{4}\right]^{2}}$

Substituting inside (3) yields

$$
\begin{aligned}
E_{n}= & E_{n}^{(0)}+\frac{\left(2 e V_{\mathrm{o}}\right)^{2}}{\hbar^{2} \pi^{2} / 2 m^{*} L^{2}} \frac{\left(\kappa_{\mathrm{D}} L / 2\right)^{2}}{\sin h^{2}\left(\kappa_{\mathrm{D}} L / 2\right)} \frac{n^{2}}{\pi^{4}} 16 \\
& \times \sum_{l \neq n} \frac{l^{2}\left(1-(-1)^{n+l}\right)^{2}}{\left[\left(n^{2}+l^{2}+\left(\kappa_{\mathrm{D}} L / \pi\right)^{2}\right)^{2}-4 n^{2} l^{2}\right]^{2}\left(n^{2}-l^{2}\right)}
\end{aligned}
$$

In the zero-shielding, infinite Debye length limit $\kappa_{\mathrm{D}} L \rightarrow$ $\infty$, when $2 V_{\mathrm{o}} \rightarrow \mathcal{E}_{\mathrm{o}} L$, one recovers the unshielded QCSE levels

$E_{n}=E_{n}^{(0)}+\frac{\left(\mathcal{E}_{\mathrm{o}} L\right)^{2}}{\hbar^{2} \pi^{2} / 2 m^{*} L^{2}} \frac{n^{2}}{\pi^{4}} 16 \sum_{l \neq n} \frac{l^{2}\left(1-(-1)^{n+l}\right)^{2}}{\left[\left(n^{2}-l^{2}\right)^{2}\right]^{2}\left(n^{2}-l^{2}\right)}$

The mode energy $E_{n}$ is always measured relative to the middle of the well; the latter always coincides with the bottom energy for the square (un-biased) QW, as shown in Fig. 1.

The shift in energy levels relative to the square QW eigen values, obtained from (7), is plotted in Fig. 2a versus the ratio $\kappa_{\mathrm{D}} L \equiv L / \lambda_{\mathrm{D}}$ for the lowest three modes. The chosen parameters are peak-to-peak sheath potential $2 V_{\mathrm{o}}$ $=50 \mathrm{meV}$, QW width $L=8 \mathrm{~nm}$ and $m_{\mathrm{e}}^{*} / m_{\mathrm{e}}=0.19$ for GaN. For $\lambda_{\mathrm{D}} \gg L / 2$ the polarization field is nearly unshielded, the potential profile nearly linear, and the red-shifting hovers near the maximum value, characterizing the ordinary QCSE. Red shifting is however reduced rapidly as the screening range becomes equal or shorter than half the QW width, $\lambda_{\mathrm{D}} \leq L / 2$, becoming completely negligible at 
$\lambda_{\mathrm{D}}<L / 4$. Beyond this point the energy levels revert to the square QW eigen values and the QCSE is completely "rectified". Using the scaling $\lambda_{\mathrm{D}}=\sqrt{4 \pi \epsilon_{\mathrm{o}} e^{2} N_{\mathrm{e}} / \kappa T}$ with the value $\epsilon_{\mathrm{o}}=8.9$ for the GaN dielectric constant recasts energy shift Fig. 2a in terms of the carrier density $N_{\mathrm{e}}$, Fig. 2b. Complete shielding of the QCSE occurs at $N_{\mathrm{e}}$ $\geq 10^{20} \mathrm{~cm}^{-3}$. This value agrees well quantitatively with similar results obtained in [17], based on the observed decrease in the radiative emission time.

As expected, perturbation theory breaks down when the polarization potential exceeds the unperturbed (square QW) energy eigen values $e V_{\mathrm{o}} \geq E_{1}^{(0)} \sim 31 \mathrm{meV}$. Since the combined inherent and strain-induced polarization fields can reach values up to $5 \mathrm{MeV} / \mathrm{cm}$ [18] and $V_{\mathrm{o}} \simeq L \mathcal{E}_{\mathrm{o}} / 2$ up to $2.5 \mathrm{~V}$ over a $10 \mathrm{~nm} \mathrm{QW}$, numerical solutions of Schrodinger Equation are required for realistic polarization values. For comparison Fig. 3 plots the lowest energy levels obtained from numerical solutions (points) and perturbation theory (curves) versus the ratio $L / 2 \lambda_{\mathrm{D}}$ for $V_{\mathrm{o}}$ $=0.250 \mathrm{~V}$. For unshielded or partially shielded QCSE with $\lambda_{\mathrm{D}} \leq L / 4$ the perturbation theory overestimates the redshift by a factor of 2 . Good agreement occurs for $\lambda_{\mathrm{D}}<L / 8$ when the charged layer thickness is much smaller than the QW thickness, and thus the size of the perturbation, parameterized by $\int_{0}^{L} \mathrm{~d} x \sin h\left(x / \lambda_{\mathrm{D}}\right) \rightarrow \lambda_{\mathrm{D}} / L$ becomes negligible.
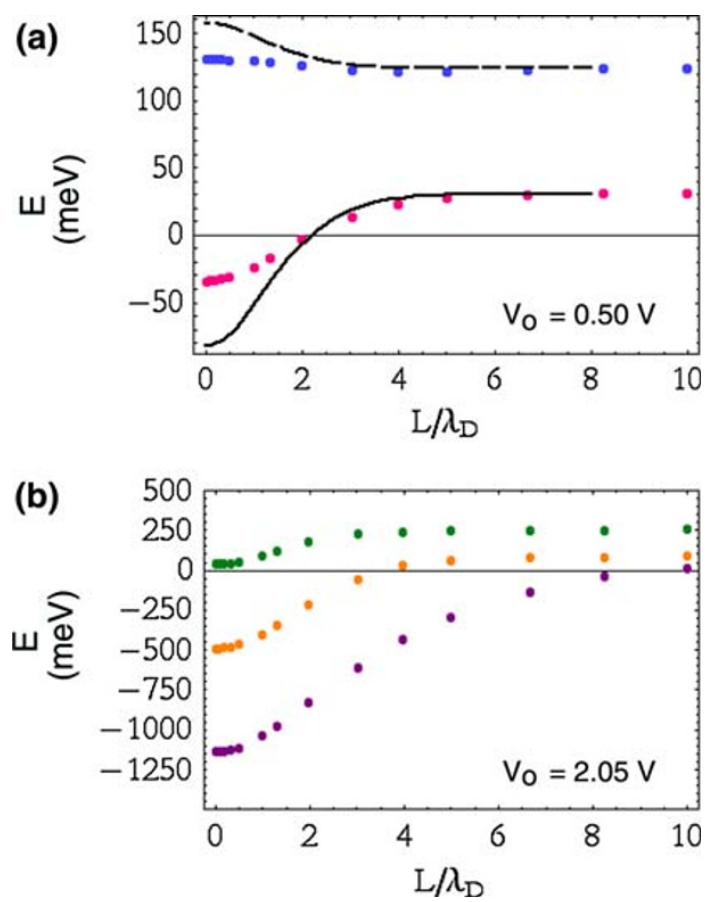

Fig. 3 a Numerical (points) and theoretical energy values (lines) for the lower two eigen modes versus $L / \lambda_{\mathrm{D}}$ for $V_{\mathrm{o}}=0.500 \mathrm{eV}$. b Numerical energy values for the lower three eigen modes versus $L / \lambda_{\mathrm{D}}$ for $V_{\mathrm{o}}=2.05 \mathrm{eV}$
It is useful, for the discussion that follows, to obtain an analytic estimate of the carrier energy eigen values for arbitrary $V_{\mathrm{o}}$ and $\lambda_{\mathrm{D}}$. To that end the eigenfunctions of Eq. 2 are obtained in terms of an infinite power series expansion a la Frobenius, Appendix 1. The fast convergence of the series solutions allows the calculation of the expectation values of the kinetic energy $\left\langle-\hbar^{2} \partial_{x}^{2} / 2 m^{*}\right\rangle$, potential energy $\langle e \Phi(x)\rangle$ and the total energy expectation value, yielding

$\left\langle E_{n}\right\rangle=\left|C_{\mathrm{o}}\right|^{2}\left[\frac{\hbar^{2}}{2 m^{*}} \frac{K_{n}}{\lambda_{\mathrm{D}}^{2}}-e V_{\mathrm{o}} W_{n}\right]$,

where $K_{n}, W_{n}$ are functions of $e V_{\mathrm{o}} / \kappa T$ and the quantum number $n$, and $C_{\mathrm{o}}$ is the wave function normalization constant. The kinetic energy $\propto 1 / \lambda_{\mathrm{D}}^{2}$ increases with decreasing $\lambda_{\mathrm{D}}$, while the potential ("edge-binding") energy is fixed. For $e V_{\mathrm{o}}>5 \kappa T$ the ratio $W_{1} / K_{1}$ for the fundamental mode is nearly constant and hovers close to $1 / 2$, Appendix 1 .

The reduction of the red shift with increasing ES shielding and decreasing shielding distance $\lambda_{\mathrm{D}}$, manifested experimentally as a blue shift relative to the unscreened QCSE, is qualitatively understood as following. For $\lambda_{\mathrm{D}}<L / 2$ the $\sin h\left(x / \lambda_{\mathrm{D}}\right)$ potential behaves like an edge-well inside the square well, instead of a tilted QW floor. If confinement within the edge-well occurs, the lowest energy level must satisfy $\left\langle E_{1}\right\rangle \leq 0$. As long as the confined "kinetic energy" $K_{1} \hbar^{2} / 2 m_{\mathrm{e}}^{*} \lambda_{\mathrm{D}}^{2}$ is less than the edge-binding energy $e V_{\mathrm{o}} W_{1}$ then $E_{1}<0$ and the wave function is trapped at the QW edge. Edge-confinement within a range shorter than the well width, $\lambda_{\mathrm{D}}<L / 2$, increases the mode energy relative to that for a tilted QW bottom and causes blue shift relative to the unshielded QCSE. The blue-shift increases with increasing carrier density, meaning shorter confinement length $\lambda_{\mathrm{D}}$. Eventually, for large enough density with $\lambda_{\mathrm{D}} \leq \sqrt{m^{*} e V_{\mathrm{o}} / \hbar^{2}}$, the kinetic energy exceeds the edgebinding energy and $\left\langle E_{1}\right\rangle>0$, edge confinement ceases, and the wave function shifts to the center to occupy the full QW width. At the same time most of the well bottom becomes nearly as flat as in a square well, since $\mathcal{E}$ is excluded from most of the interior. Full "rectification" of the QCSE occurs and the eigen values and eigen modes approach that of a square QW.

Transition from edge-confinement to full QW occupation occurs for either $V_{\mathrm{o}}<V_{\text {th }}$ or $\lambda_{\mathrm{D}} \leq \lambda_{\text {th }}$, where $V_{\text {th }} \equiv \hbar^{2} / e m_{\mathrm{e}}^{*} \lambda_{\mathrm{D}}^{2}$ is the threshold under given $\lambda_{\mathrm{D}}$, and $\lambda_{\text {th }} \equiv$ $\hbar / \sqrt{e V_{\mathrm{o}} m_{\mathrm{e}}^{*}}$ the threshold under given $V_{\mathrm{o}}$. This transition is shown in Fig. $4 \mathrm{a}$ and $\mathrm{b}$, plotting the fundamental mode profiles $\Psi(x)$ for various values of $\lambda_{\mathrm{D}} / L$, for low and high voltages, respectively $V_{\mathrm{o}}=0.250 \mathrm{~V}$ and $V_{\mathrm{o}}=2.05 \mathrm{~V}$. As the screening distance decreases, the center of the wave function moves from the left edge towards the center of the 

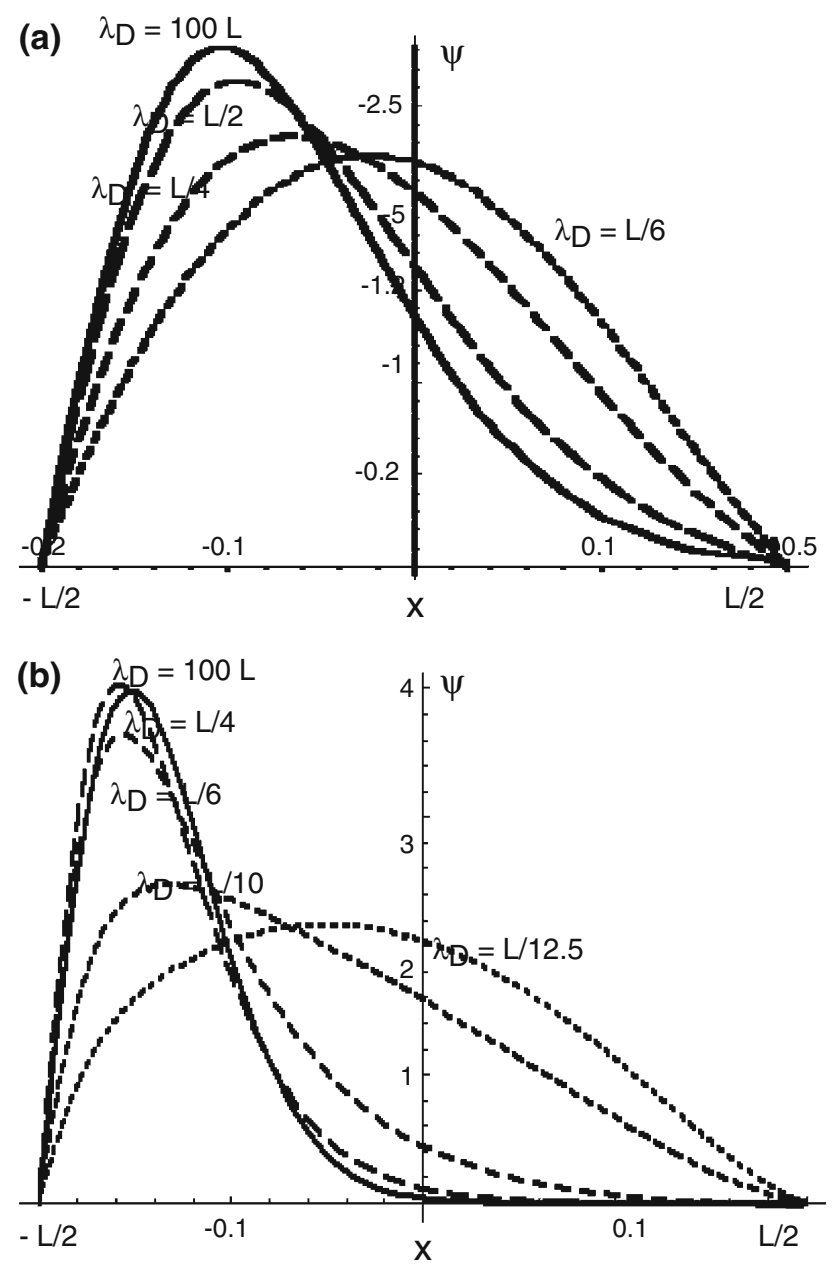

Fig. 4 Normalized wave function profiles (a.u.) for various values $\lambda_{\mathrm{D}} / L$ as marked and for: $\mathbf{a} V_{\mathrm{o}}=0.25 \mathrm{eV} \mathrm{b} V_{\mathrm{o}}=2.05 \mathrm{eV}$. Transition from edge-trapping to full $\mathrm{QW}$ occupation occurs at shorter $\lambda_{\mathrm{D}}$ (higher carrier density) for higher polarization voltage

well. The transition to full QW occupancy occurs at shorter screening length $\lambda_{\mathrm{D}}$ for higher $V_{\mathrm{o}}$ (Fig. 4b).

Figure 5a plots the lower two eigen values versus sheath potential, for given $\lambda_{\mathrm{D}}=L / 8$. The fundamental $E_{1}$ becomes positive at about $V_{\mathrm{o}} \simeq V_{\text {th }} \equiv \hbar^{2} / e m_{\mathrm{e}}^{*} \lambda_{\mathrm{D}}^{2}$. For $V_{\mathrm{o}}<V_{\text {th }}$ the value $E_{1}$ increases and tends to the square well limit as $V_{\mathrm{o}} \simeq 0$. Figure $5 \mathrm{~b}$ shows the fundamental eigen value $E_{1}$ versus $L / \lambda_{\mathrm{D}}$ for two different voltages $V_{\mathrm{o}}$. The eigen values asymptote to the square QW limit at shorter screening distance for the case of higher polarization $V_{\mathrm{o}}$.

\section{Radiative Emission Probability}

The changes in the wave function profiles have a profound influence in the $e-h$ transition probability during radiative emission, proportional to the dipole moment overlap integral
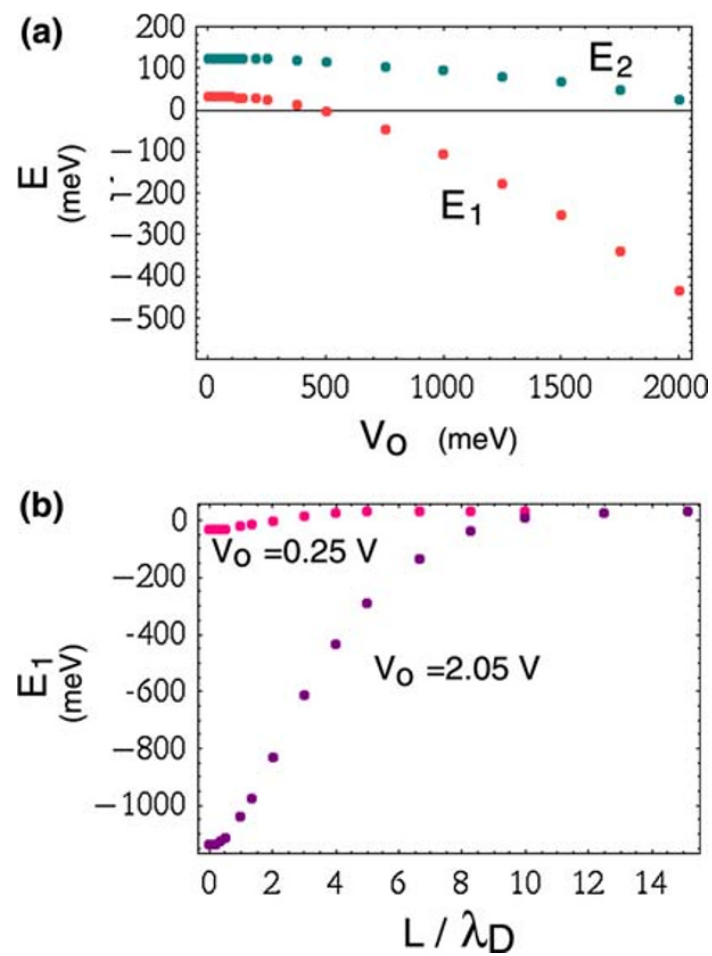

Fig. 5 a Energy levels for the lower two eigen modes versus $V_{\mathrm{o}}$ for fixed $\lambda_{\mathrm{D}}=L / 8$ b Fundamental level versus $L / \lambda_{\mathrm{D}}$ for two polarization voltages $V_{\mathrm{o}}=0.250 \mathrm{~V}$ and $V_{\mathrm{o}}=2.05 \mathrm{~V}$, corresponding to polarization values $\mathcal{E}_{\mathrm{o}}=0.65 \mathrm{MV} / \mathrm{cm}$ and $\mathcal{E}_{\mathrm{o}}=5.01 \mathrm{MV} / \mathrm{cm}$ respectively

$\mathbf{p}_{\mathbf{e h}}=\int \mathrm{d} \mathbf{r}^{3} \Psi_{h}^{*}(\mathbf{r}) u_{v}^{*}(\mathbf{r}) \nabla_{\mathbf{r}} \Psi_{e}(\mathbf{r}) u_{c}(\mathbf{r})$

where $u_{c}(\mathbf{r}), u_{v}(\mathbf{r})$ are the lattice-periodic parts and $\Psi_{e}(\mathbf{r}), \Psi_{h}(\mathbf{r})$ the slowly varying envelope functions obtained from (2). Employing, as usual, the space-scale separation between the rapidly varying, on the latticeconstant scale, $u_{c}, u_{v}$, and the slowly varying envelopes, valid for as long as $L, \lambda_{\mathrm{D}} \gg a$, the above is approximated by

$$
\begin{aligned}
\mathbf{p}_{e h} \simeq & \int_{-L / 2}^{L / 2} \mathrm{~d} x \psi_{h}^{*}(x) \psi_{e}(x) \iint \mathrm{d} y \mathrm{~d} z \mathrm{e}^{\mathrm{i} k_{x}^{e} x-\mathrm{i} k_{x}^{h} x} \mathrm{e}^{\mathrm{i} k_{y}^{e} x-i k_{y}^{h} x} \\
& \times \int_{C} \mathrm{~d} \mathbf{r}^{3} u_{v}(\mathbf{r}) \nabla_{\mathbf{r}} u_{c}(\mathbf{r}) .
\end{aligned}
$$

Orthogonality among the lattice functions $u_{c}, u_{v}$ was used in arriving at (10). The last integral over the unit lattice unit cell volume $C$ is independent of the polarization. For "vertical transitions" with $\mathbf{k}_{e}-\mathbf{k}_{h}=\mathbf{k}_{p} \simeq \mathbf{0}$ (given that $\left.k_{p}=\omega / c \ll\left|k_{e, h}\right|\right)$ the dependence on the polarization voltage $V_{\mathrm{o}}$ and screening distance $\lambda_{\mathrm{D}}$ is carried entirely in the overlapping between electron-hole envelopes

$\mathbf{p}_{e h}=\mathbf{G} \int_{-L / 2}^{L / 2} \mathrm{~d} x \psi_{h}^{*}\left(x ; V_{\mathrm{o}}, \lambda_{\mathrm{D}}\right) \psi_{\mathrm{e}}\left(x ; V_{\mathrm{o}}, \lambda_{\mathrm{D}}\right)$

with $\mathbf{G} \equiv \int_{C} d \mathbf{r}^{\mathbf{3}} u_{v}(\mathbf{r}) \nabla_{\mathbf{r}} u_{c}(\mathbf{r})$ a constant. Here we will assume, due to the symmetry in the sinh potential, that 
$\Psi_{h}(x)=\Psi_{e}(L-x)$. Taking the transition probability for a flat QW with $\psi_{e, h}\left(x ; V_{\mathrm{o}}=0, \lambda_{\mathrm{D}}=\infty\right)=\cos (\pi x / L) / \sqrt{L}$ as reference, and since the emission time $\tau \propto 1 / \mathbf{p}_{e h}^{2}$, one has

$$
\begin{aligned}
\frac{\tau^{-1}}{\tau_{\mathrm{o}}^{-1}} & =\frac{\left[\int_{-L / 2}^{L / 2} \mathrm{~d} x \psi_{h}^{*}\left(x ; V_{\mathrm{o}}, \lambda_{\mathrm{D}}\right) \psi_{e}\left(x ; V_{\mathrm{o}}, \lambda_{\mathrm{D}}\right)\right]^{2}}{\left[\int_{-L / 2}^{L / 2} \mathrm{~d} x \cos (\pi x / L)^{2} / L\right]^{2}} \\
& =\left[\int_{-L / 2}^{L / 2} \mathrm{~d} x \psi^{*}\left(x-L ; V_{\mathrm{o}}, \lambda_{\mathrm{D}}\right) \psi\left(x ; V_{\mathrm{o}}, \lambda_{\mathrm{D}}\right)\right]^{2}
\end{aligned}
$$

The ratio $\tau_{\mathrm{o}} / \tau$ is potted in Fig. 6a versus $L / \lambda_{\mathrm{D}}$ for various peak voltages $V_{\mathrm{o}}$, using the wave function profiles obtained from numerical solutions. Characteristic emission times tend to increase with increasing applied polarization voltage $V_{\mathrm{o}}$, and decrease with decreasing screening distance $\lambda_{\mathrm{D}}$. The results of Fig. $6 \mathrm{a}$ are plotted verusus the corresponding carrier density $N$ in Fig. $6 \mathrm{~b}$, for QW width $8 \mathrm{~nm}$. These results reproduce the three order of magnitude emission increase between densities $10^{19}$ and $10^{21}$, resulting in complete rectification of the QCSE, that was first obtained using detailed Poisson-Schrodinger simulations in Ref. [17] for a $7 \mathrm{~nm}$ QW.

A careful comparison between the energy blue-shifting with increasing density (screening), Fig. 7a, and the decrease in recombination time, Fig. $7 \mathrm{~b}$, shows that the rectification of the QCSE red-shift occurs before the
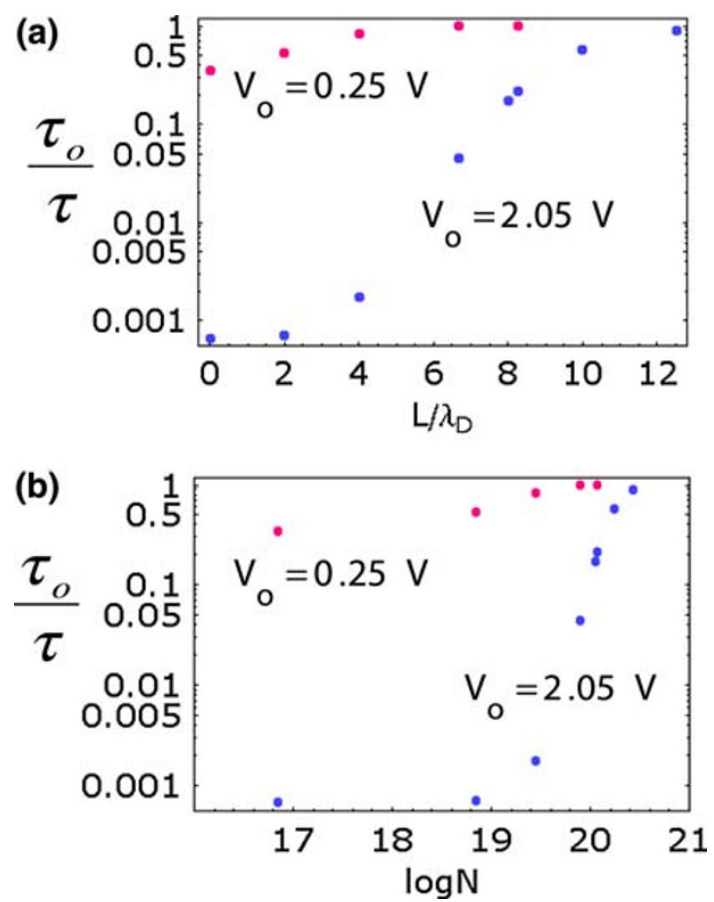

Fig. 6 a Ratio of radiative emission time for a flat QW to that of the ES-shielded QCSE versus screening distance $L / \lambda_{\mathrm{D}}$, for low and high polarization voltages b same plotted versus corresponding carrier density $N$ for an $8 \mathrm{~nm}$ QW
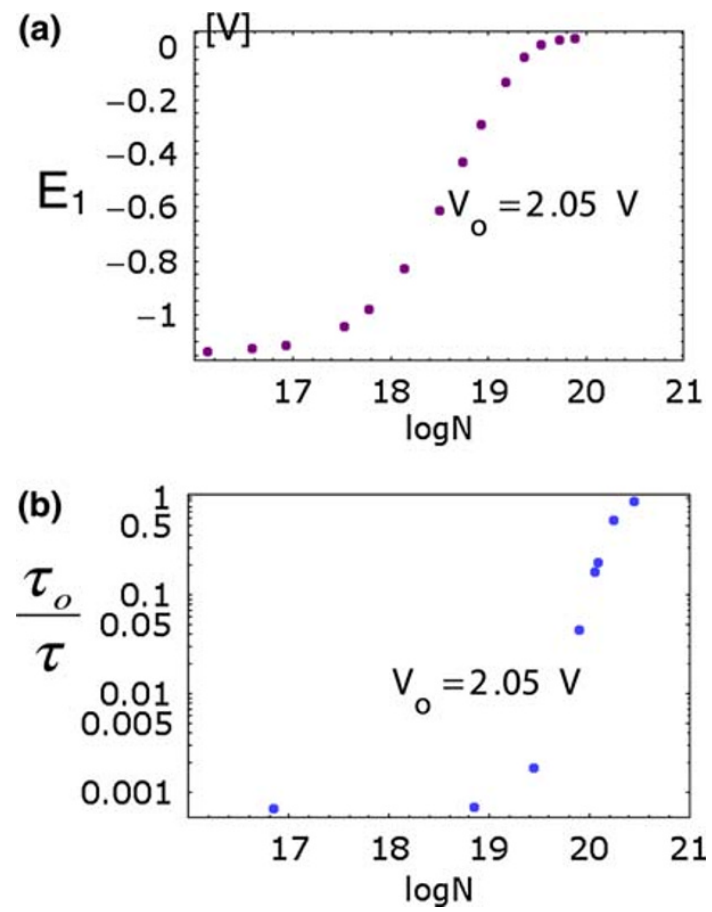

Fig. 7 Comparative evolution of a lowest confined mode energy and b recombination time versus carrier density $N$, for an $8 \mathrm{~nm}$ thickness QW

recovery of the radiative emission time: the energy redshifting is gradually eliminated first, between densities $10^{17} \mathrm{~cm}^{-3}$ and $10^{19} \mathrm{~cm}^{-3}$, though the radiative emission time remains almost constant there. The emission probability is restored, rather abruptly, at higher densities between $10^{19} \mathrm{~cm}^{-3}$ and $10^{20} \mathrm{~cm}^{-3}$. This lagging in restoring the emission probability is explained via edge-carrier trapping, mentioned in the previous discussion. As carrier density increases and the edge-potential range $\lambda_{\mathrm{D}}$ narrows down, the increasing edge-confinement of the wave function causes the energy level $E_{1} \propto \hbar^{2} / 2 m^{*} \lambda_{\mathrm{D}}^{2}$ to increase. As long as the "confinement energy" $\hbar^{2} / 2 m^{*} \lambda_{\mathrm{D}}^{2}$ is smaller than the edge potential depth $e V_{\mathrm{o}}$ electron and hole wave functions remain edge-localized and no significant change in overlap and in recombination time occurs. The abrupt decrease in the radiative emission time (increase in the radiative emission rate) occurs after $\hbar^{2} / 2 m^{*} \lambda_{\mathrm{D}}^{2} \geq e V_{\mathrm{o}}$, since at this point the wave function moves from edge-confinement to full $\mathrm{QW}$ occupancy. Practically this means that the QCSE-related energy red-shift has already been eliminated before the radiative emission time recovers. This behavior agrees with the results in [17].

\section{Shielding of the Peak Polarization Voltage}

It has so far been tacitly assumed that the charged layer peak-voltage $V_{\mathrm{o}}$ is independent of the screening carrier 
density $N_{e, h}$ and the peak-to-peak voltage $2 V_{\mathrm{o}}$ was taken equal to the "polarization voltage" $V_{\mathrm{p}} \equiv \mathcal{E}_{\mathrm{o}} L$ for an unscreened QW, Fig. 2a. In other words the shielding only modified the potential profile across the QW. However, for given applied $\mathcal{E}_{\mathrm{o}}$ and $L$, the shielded $V_{\mathrm{o}}$ does depend on the carrier density, and in fact $V_{\mathrm{o}}$ is reduced below $V_{\mathrm{p}}$ at high carrier densities. The shielding of the peak voltage is summarized below, based on results from earlier studies [15].

Self-consistent charged layer solutions under FermiDirac thermodynamic equilibrium [15] show that as the QW thickness $L$ increases well beyond $\lambda_{\mathrm{D}}$ the peak-to-peak voltage asymptotes rapidly to a maximum saturation value $V_{\mathrm{s}}\left(\mathcal{E}_{\mathrm{o}}, N\right)$. Figure 8 a plots $2 V_{\mathrm{o}}$ versus $L$ for various polarization strength values and shows the saturation $2 V_{\mathrm{o}} \rightarrow$ $V_{\mathrm{s}}=$ constant for $L / \lambda_{\mathrm{D}} \gg 1$. Clearly $V_{\mathrm{s}}$ increases with polarization strength $\mathcal{E}_{\mathrm{o}}$. The dependence of $V_{\mathrm{s}}$ on density is given in Fig. 8b. The fact that $V_{\mathrm{s}}$ decreases with increasing density stems from Gausses law: it takes a given amount of surface charge $4 \pi \sigma \equiv e N_{\mathrm{o}} \delta L=\mathcal{E}_{\mathrm{o}}$ to screen a given field. Applying scaling arguments the charge layer thickness is $\delta L \sim\left(\mathcal{E}_{\mathrm{o}} / 2\right) / 4 \pi e N_{\mathrm{o}}$ (half of the electric field screened at each QW edge) and the sheath voltage $e V_{\mathrm{o}} \sim 4 \pi e N_{\mathrm{o}} \delta L^{2} / 2=\left(\mathcal{E}_{\mathrm{o}}^{2} / 4\right) / 2\left(4 \pi e N_{\mathrm{o}}\right)=\lambda_{\mathrm{D}}^{2} e^{2} \mathcal{E}_{\mathrm{o}}^{2} / 8(\kappa T)$.

Thus for given polarization $\mathcal{E}_{\mathrm{o}}$ the voltage $V_{\mathrm{s}}$ scales roughly as $\lambda_{\mathrm{D}}^{2} \propto 1 / N_{\mathrm{o}}$ when $L>2 \lambda_{D}$.

The screened voltage value is always less or equal to the intrinsic "polarization voltage", $2 V_{\mathrm{o}} \leq V_{\mathrm{s}} \leq V_{\mathrm{p}} \equiv \mathcal{E}_{\mathrm{o}} L$. This is shown in Fig. 8c, plotting the ratio of the peak-topeak voltage $2 V_{\mathrm{o}}$ to $V_{\mathrm{p}}$, versus sheath length, for given doping density $N_{\mathrm{D}}=10^{18} \mathrm{~cm}^{-3}$. For as long as $L \leq 2 \lambda_{\mathrm{D}}$ one has unsaturated behavior $2 V_{\mathrm{o}} \simeq V_{\mathrm{p}} \propto L$. Once saturation is reached for $L>2 \lambda_{\mathrm{D}}$ the peak-to-peak voltage is pinned at $V_{\mathrm{s}}$, independent of $L$. This is because when $L>2 \lambda_{\mathrm{D}}$ the polarization field is screened-out from the QW interior length $L-2 \lambda_{\mathrm{D}}$ that yields a negligible contribution to the voltage difference; $V_{\mathrm{s}}$ comes entirely from two charged layers of width $\lambda_{\mathrm{D}}$. Hence, for wide QWs the peakto-peak voltage turns out much smaller than the polarization voltage, and the ratio $2 V_{\mathrm{o}} / V_{\mathrm{p}}$ goes as $1 / L$. Notice that the saturation length $L_{\mathrm{s}}$ where $2 V_{\mathrm{o}}$ dips below $V_{\mathrm{p}}$ depends also on the field strength; letting $L_{\mathrm{s}} \simeq \lambda_{\mathrm{D}}$ and $V_{\mathrm{s}}=$ $L_{\mathrm{s}}^{2} e^{2} \mathcal{E}_{\mathrm{o}}^{2} / 8(\kappa T)=V_{\mathrm{p}}=\mathcal{E}_{\mathrm{o}} L_{\mathrm{s}}$ yields $L_{\mathrm{s}}=8 \kappa T / \mathcal{E}_{\mathrm{o}}$, thus saturation occurs at smaller QW thickness with increasing $\mathcal{E}_{\mathrm{o}}$. According to Fig. 8c, one may apply unsaturated values $2 V_{\mathrm{o}} \simeq V_{\mathrm{p}}$ for $\mathrm{QW}$ thickness $L<10 \mathrm{~nm}$ and for $\mathcal{E}_{\mathrm{o}} \leq 3 \mathrm{MV} / \mathrm{cm}$, up to doping densities $10^{19} \mathrm{~cm}^{-3}$. This is illustrated in Fig. 9, plotting the ratio $2 V_{\mathrm{o}} / V_{\mathrm{p}}$ versus doping density $N_{\mathrm{D}}$ for fixed $\mathrm{QW} L=8 \mathrm{~nm}$ and for various strengths $\mathcal{E}_{\mathrm{o}}$.

For given $L=8 \mathrm{~nm}$, the values $2 V_{\mathrm{o}}$ assume their saturation values and the shielded voltage falls significantly below $V_{\mathrm{p}}$ when doping densities exceed $\geq 10^{20} \mathrm{~cm}^{-3}$. This
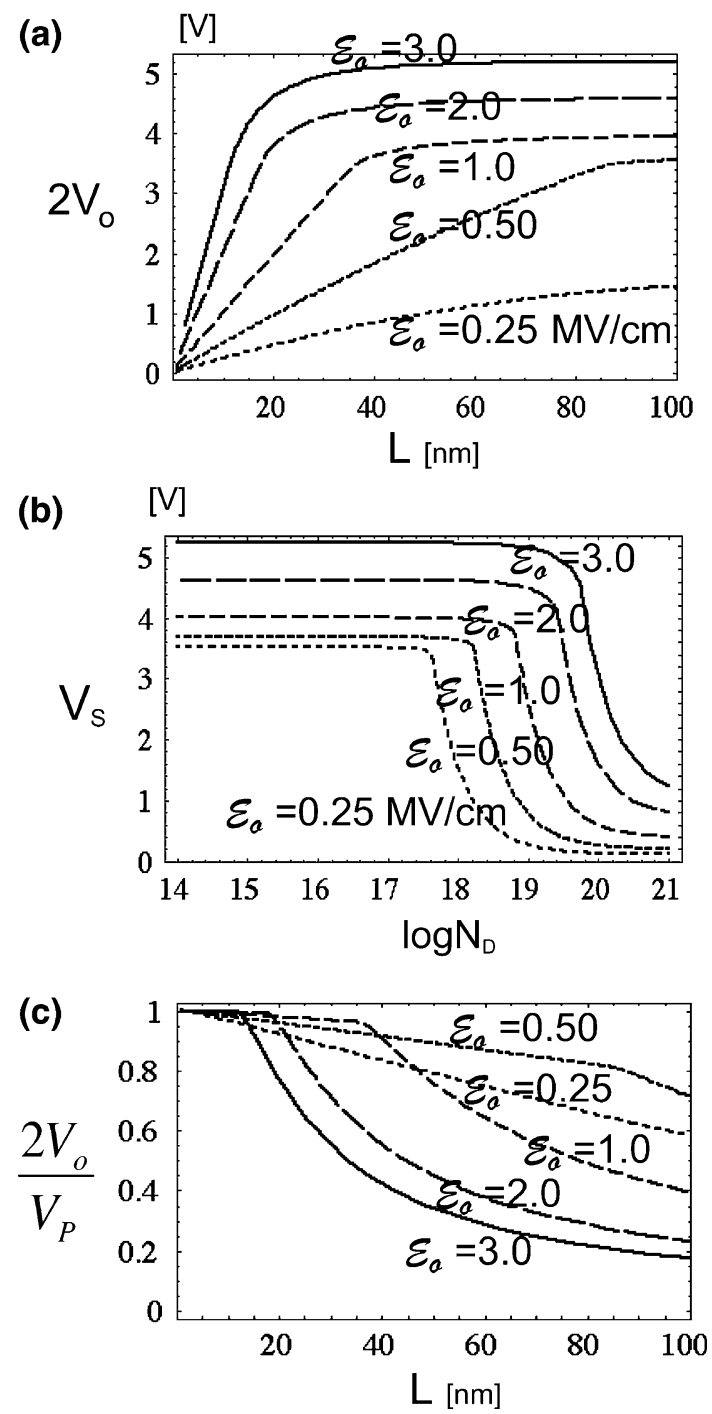

Fig. 8 Carrier density effects on the shielded voltage. a peak-to-peak voltage versus QW thickness for doping density $N_{\mathrm{D}}=10^{18} \mathrm{~cm}^{-3}$ and various polarization strengths, as marked $\mathbf{b}$ Saturated peak-to-peak voltage versus doping density $N_{\mathrm{D}}$ for various polarization strengths c ratio of peak voltage to the polarization potential versus $\mathrm{QW}$ thickness for doping density $N_{\mathrm{D}}=10^{18} \mathrm{~cm}^{-3}$

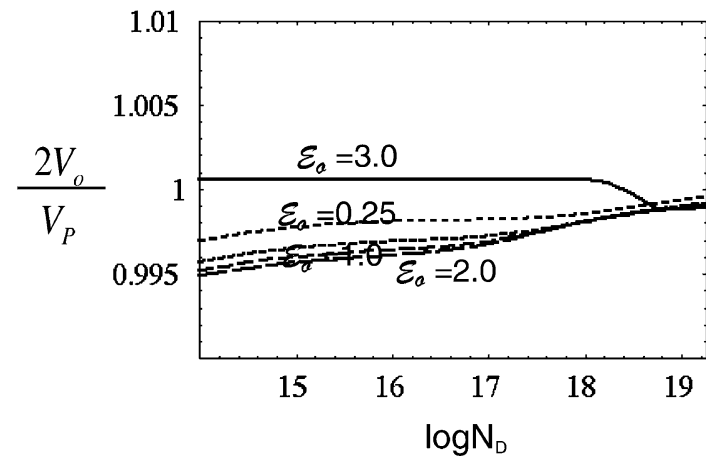

Fig. 9 ratio of peak voltage to the polarization potential versus doping density $N_{\mathrm{D}}$ in a QW of thickness $L=8 \mathrm{~nm}$, for various polarization strengths 
is illustrated in Fig. 10, showing the screened potential profiles, 10a, and electric fields, $10 \mathrm{~b}$, for various doping levels $N_{\mathrm{D}}$ across an $8-\mathrm{nm} \mathrm{QW}$ for $\mathcal{E}_{\mathrm{o}}=0.7 \mathrm{MV} / \mathrm{cm}$. The peak-to-peak voltage decreases well below $V_{\mathrm{p}}$ with increasing $N_{\mathrm{D}}$. In addition, the electron and hole charged layers become asymmetric: $V_{\mathrm{e}}$ across the negative charged layer is different than $V_{\mathrm{h}}$ across the positive charged layer. In general, reduction of the peak-to-peak voltage, as well as asymmetric electron-hole profiles should be considered for a more accurate description of the ES shielded QCSE. In particular, the drop in $V_{\mathrm{s}}<V_{\mathrm{p}}$ with increasing density could accelerate the cancellation of the QCSE and the blue shifting of the energy levels. For the relevant to our GaN experiments parameters, however, the red-shifting is all but cancelled out at density $10^{19} \mathrm{~cm}^{-3}$, just before such effects become significant. Thus it appears that energy level blueshifting caused by the $\sin h$ effect in the potential profile cancels to a large degree the QCSE effect, before shielding of the peak amplitude itself becomes important.
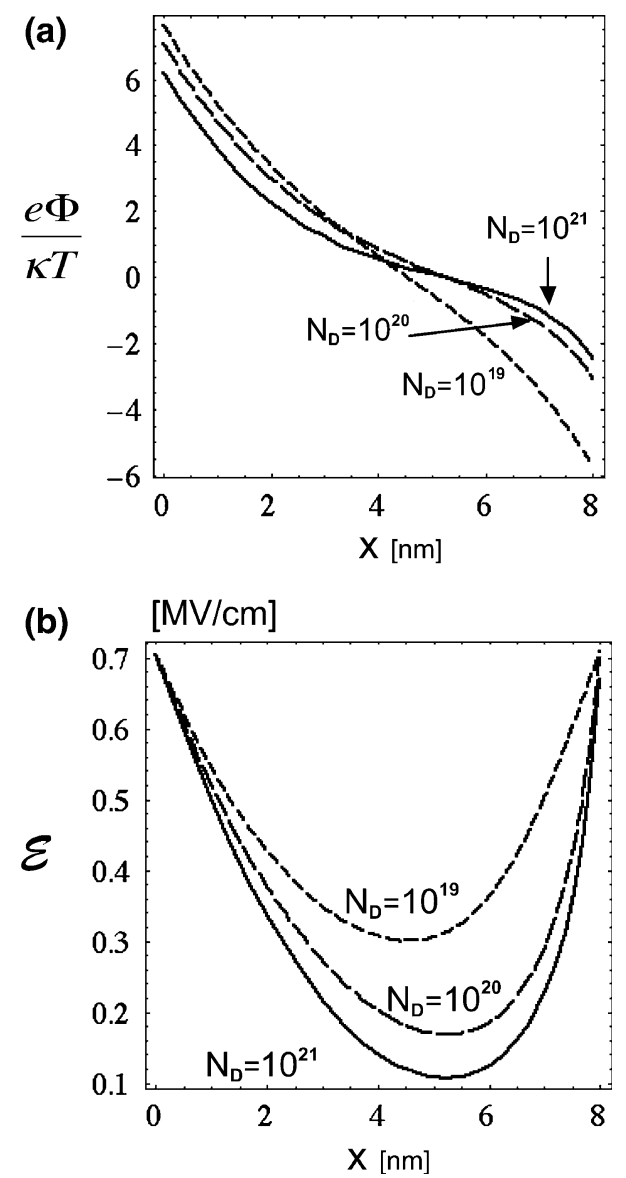

Fig. 10 a Self-consistent shielded potential profiles across an $L=8 \mathrm{~nm}$ QW for intrinsic polarization field $\mathcal{E}_{\mathrm{o}}=0.7 \mathrm{MV} / \mathrm{cm}$, for various carrier densities as marked. b Corresponding shielded electric field profiles

\section{Conclusions}

A simplified model employing ES-shielded, exponentiallydecaying polarization potentials localized at the QW edges, was employed to study the QCSE at high doping densities. Blue shifting of energy levels relative to the unshielded QCSE occurs with increasing carrier density, due to the wave function constriction within scale length $\lambda_{\mathrm{D}}<L / 2$. When the "edge-localization energy" $\hbar^{2} / m^{*} \lambda_{\mathrm{D}}^{2}$ exceeds the peak-voltage of the charged layer $e V_{\mathrm{o}}$ the wave function center shifts to the middle of the QW and behavior becomes similar to that of a square (unbiased) QW. In addition, at very high doping the shielded peak voltage is reduced well below the original unshielded "polarization voltage" $V_{\mathrm{p}}$. Both effects cause gradual elimination of the QCSE redshifting, an increase in the $e-h$ wave function overlap and a decrease of the radiative emission time. A significant reduction of the peak polarization voltage requires higher carrier densities than most practical situations, and screening effects stem mainly from the interior-screening and the localization of the polarization voltage within QW edgelayers. Our theoretical estimates show that the elimination of the QCSE related red-shift in energy precedes the recovery in the radiative emission time, in quantitative agreement with experimental measurements in [17].

\section{Appendix-1: 1-D Edge-confined Modes-Asymptotic Polynomial Expansions}

Section "QW Eigen Modes with ES-shielded Polar Potential" derived a perturbative solution for the edgeconfined modes in terms of the square well eigen modes. Another approach, involving an infinite series polynomial expansion, will be given here and used to derive the scaling of the edge-confined expectation values for the kinetic and potential energy. First, for $\lambda_{\mathrm{D}} \ll L / 2$ one may approximate the sinh potential for $x<0, \quad \Phi=-V_{\mathrm{o}} \sin h\left[|x| / \lambda_{\mathrm{D}}\right] /$ $\sin h\left[L / 2 \lambda_{\mathrm{D}}\right]$, as $-V_{\mathrm{o}} \exp \left[(|x|-L / 2) / \lambda_{\mathrm{D}}+L / 2 \lambda_{\mathrm{D}}\right] / \exp [L /$ $\left.2 \lambda_{\mathrm{D}}\right]=-V_{\mathrm{o}} \exp \left[-\zeta / \lambda_{\mathrm{D}}\right]$ where $\zeta$ the distance from the edge $\zeta \equiv L / 2-|x|$. The $\sin h$ Schrodinger Equation 2 is then approximated by one for an exponential potential $-e V_{\mathrm{o}} \exp \left[-\zeta / \lambda_{\mathrm{D}}\right]$ which has been analyzed elsewhere. ${ }^{1} \mathrm{~A}$ dimensionless scaling measuring length in units of $\lambda_{\mathrm{D}}$ and energy in units of $\hbar^{2} / 2 m \lambda_{\mathrm{D}}^{2}$ yields

$-\frac{d^{2}}{\mathrm{~d} \bar{\zeta}^{2}} \psi_{n}-\bar{V}_{\mathrm{o}} e^{-\bar{\zeta}} \psi_{n}=-\varepsilon_{n} \psi_{n}$

where $n$ labels the energy quantum number $\bar{E}_{n} \equiv-\bar{\varepsilon}_{n}$. A change of variable $w=e^{-\bar{\zeta}}$ for $\bar{\zeta}>0$ with $\mathrm{d} \psi / \mathrm{d} \bar{\zeta}=$

\footnotetext{
1 The solutions with $\Psi(-L / 2)=\Psi(\zeta=0)=0$ are the odd-symmetry eigenfunctions of the general attractive potential $-e V_{\mathrm{o}} \exp \left[-|\zeta| / \lambda_{\mathrm{D}}\right]$.
} 
$-w \mathrm{~d} \psi / \mathrm{d} w$ removes the exponential term and reduces (13) to

$w^{2} \frac{d^{2}}{\mathrm{~d} w^{2}} \psi_{n}+w \frac{d}{\mathrm{~d} w} \psi_{n}+\bar{V}_{\mathrm{o}} w \psi_{n}-\bar{\varepsilon}_{n} \psi_{n}=0$.

The boundary conditions at $\bar{\zeta}=0, \infty$ correspond to $w=1$, 0 , and are given by $\psi_{\bar{\zeta}=\infty}=\psi_{w=0}=0$. A series expansion

$\psi_{n}=w^{\xi} \sum_{l=0}^{\infty} c_{l} w^{l}$

inside (14) yields the coefficient recurrence relation $c_{l+1}=$ $c_{l}\left(-\bar{V}_{\mathrm{o}}\right) /(l+2 \xi)$, or ,

$c_{l}=c_{\mathrm{o}} \frac{\left(-\bar{V}_{\mathrm{o}}\right)^{l}}{(1+2 \xi)(2+2 \xi) \cdots(l+2 \xi)}=c_{\mathrm{o}}\left(-\bar{V}_{\mathrm{o}}\right)^{l} \frac{l !}{(l+2 \xi) !}$

where $\quad(l+2 \xi) ! \equiv(1+2 \xi)(2+2 \xi) \cdots(l+2 \xi)=$ $\Gamma(l+2 \xi) / \Gamma(2 \xi)$ and $c_{\mathrm{o}}^{n}$ is found from the normalization condition. Substitution into the series solution and application of the boundary conditions at $w=1(\bar{\zeta}=0)$ yields the eigen values $\xi=+\sqrt{\bar{\varepsilon}_{n}}$ from the roots of the following indicial equation

$1+\sum_{l=1}^{\infty} \frac{\left(-\bar{V}_{\mathrm{o}}\right)^{l}}{l !(l+2 \xi) !}=0$.

Switching (15) back to the original variables yields the corresponding eigenfunctions as

$$
\begin{aligned}
\psi_{n}(\zeta) & =\sum_{l=0}^{\infty} c_{l}^{m} e^{-\left(l+\xi_{n}\right) \zeta / \lambda_{D}} \\
& =\sum_{l=0}^{\infty} c_{\mathrm{o}}\left(-\bar{V}_{\mathrm{o}}\right)^{l} \frac{l !}{\left(l+2 \xi_{n}\right) !} e^{-\left(l+\xi_{n}\right) \zeta / \lambda_{\mathrm{D}}}
\end{aligned}
$$

making use of $\xi=+\sqrt{\bar{\varepsilon}_{n}}$. The leading term goes as $\exp \left[-\sqrt{\bar{\varepsilon}_{n}} \zeta / \lambda_{\mathrm{D}}\right]$ and gives the asymptotic behavior at $|\zeta| \gg$ $\lambda_{\mathrm{D}}$. For practical purposes is suffices to keep polynomial terms up to order $M$ equal to twice the integer part $\left[\bar{V}_{\mathrm{o}}\right]$ inside the infinite sum in (17).

One may now compute expectation values with direct integration of (18). First, orthonormalization $\int_{0}^{\infty} \mathrm{d} \zeta \Psi^{*} \Psi=$ 1 yields the normalization constant $c_{\mathrm{o}}$ from

$\lambda_{\mathrm{D}}\left|c_{\mathrm{o}}\right|^{2} \sum_{l=0}^{\infty} \sum_{k=0}^{\infty} \frac{\left(-\bar{V}_{\mathrm{o}}\right)^{l+k}}{l+k+2 \xi_{n}} \frac{l ! k !}{\left(l+2 \xi_{n}\right) !\left(k+2 \xi_{n}\right) !}=1$

The expectation potential energy $\langle e V\rangle=$ $-\int_{0}^{\infty} \mathrm{d} \zeta e V_{\mathrm{o}} e^{-\zeta / \lambda_{\mathrm{D}}} \Psi^{*} \Psi$ yields $\langle V\rangle=\lambda_{\mathrm{D}}\left|c_{\mathrm{o}}\right|^{2} e V_{\mathrm{o}} W$ with

$W_{n}=\sum_{l=0}^{\infty} \sum_{k=0}^{\infty} \frac{\left(-\bar{V}_{\mathrm{o}}\right)^{l+k}}{l+k+2 \xi_{n}+1} \frac{l ! k !}{\left(l+2 \xi_{n}\right) !\left(k+2 \xi_{n}\right) !}$

and the expectation kinetic energy $\left\langle\mathcal{K}_{n}\right\rangle=-\left(\hbar^{2} / 2 m^{*}\right)$ $\int_{0}^{\infty} \mathrm{d} \zeta \Psi^{*} \frac{d}{\mathrm{~d} \zeta^{2}} \Psi$ yields $\left\langle\mathcal{K}_{n}\right\rangle=\lambda_{\mathrm{D}}\left|c_{\mathrm{o}}\right|^{2}\left(\hbar^{2} / 2 m^{*}\right) K_{n} / \lambda_{\mathrm{D}}^{2}$

$$
\begin{aligned}
K_{n}=- & \sum_{l=0}^{\infty} \sum_{k=0}^{\infty} \frac{\left(-\bar{V}_{\mathrm{o}}\right)^{l+k}\left(l+2 \xi_{n}\right)\left(l+2 \xi_{n}+1\right)}{l+k+2 \xi_{n}+2} \\
& \times \frac{l ! k !}{\left(l+2 \xi_{n}\right) !\left(k+2 \xi_{n}\right) !}
\end{aligned}
$$

Thus the energy expectation value $\left\langle E_{n}\right\rangle$ is

$\left\langle E_{n}\right\rangle=\lambda_{\mathrm{D}}\left|c_{\mathrm{o}}\right|^{2}\left[\frac{\hbar^{2}}{2 m^{*}} \frac{K_{n}}{\lambda_{\mathrm{D}}^{2}}-e V_{\mathrm{o}} W_{n}\right]$

where the normalization factor $\left|c_{\mathrm{o}}\right|^{2} \lambda_{\mathrm{D}} \equiv\left|C_{\mathrm{o}}\right|^{2} \sim 1$ from (19). Thus edge detrapping at about $\left\langle E_{1}\right\rangle>0$ occurs for $\lambda_{\mathrm{D}}^{2} \leq\left(\hbar^{2} / 2 m^{*} e V_{\mathrm{o}}\right) /\left(W_{1} / K_{1}\right)$. Both $K$ and $W$ depend on $\bar{V}_{\mathrm{o}}$ and on the energy eigen value $-\varepsilon_{1}$ where $\varepsilon_{1}=\xi_{1}^{2}$. The ratio $W_{1} / K_{1}$ is plotted in Fig. 11 versus the peak voltage $\bar{V}_{\mathrm{o}}$ (normalized in units of $\kappa T$ ) using the lowest mode energy $n=1$ inside (20) and (21). Note that for $V_{\mathrm{o}}>5 \kappa T$ the ratio hovers near $1 / 2$ and thus detrapping occurs at $\lambda_{\mathrm{D}} \leq \hbar / \sqrt{m^{*} e V_{\mathrm{o}}}$.

\section{Appendix 2: Charged Layer Potential}

The self-consistent Poisson's equation, including the influence of the charged layer (plasma sheath) potential $\Phi(x)$ on the Fermi-Dirac occupation number $f$ in determining the local carrier density is

$\frac{d^{2}}{\mathrm{~d} x^{2}} \Phi=-\rho(\Phi[x])$,

subject to the boundary conditions $-d \Phi /\left.\mathrm{d} x\right|_{x=-L / 2}=$ $-d \Phi /\left.\mathrm{d} x\right|_{x=L / 2}=\mathcal{E}_{\mathrm{o}}$. This means that $\mathcal{E}(x)$

equals the unshielded value at each QW edge. Above we have normalized $\Phi \rightarrow e \Phi / \kappa T, x \rightarrow x / \lambda_{\mathrm{D}}$ and $\rho \rightarrow \rho / e N_{\mathrm{o}}$ where $N_{\mathrm{o}}$ is a reference carrier density and $\lambda_{\mathrm{D}}=$ $\sqrt{\kappa T \epsilon / 4 \pi e^{2} N_{\mathrm{o}}}$ the corresponding Debye length which includes the dielectric shielding $\varepsilon$ from core (bound) electrons. The sum of the electron, hole and charged donor charge densities ( $\mathrm{N}$-doping is assumed without loss of

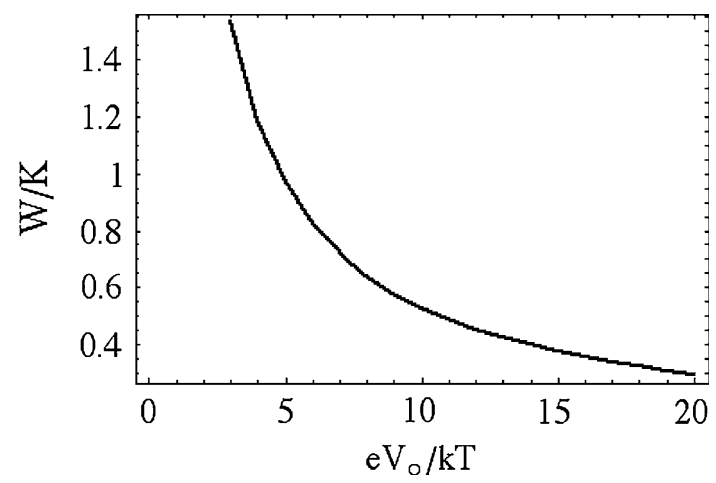

Fig. 11 Ratio of $W_{1} / K_{1}$ versus peak-voltage 
generality) on the right-hand side follows from the equilibrium Fermi-Dirac occupation numbers,

$$
\begin{aligned}
\rho(x)= & -\int_{E_{C}}^{\infty} \mathrm{d} E \frac{G_{\mathrm{e}}(E)}{1+e^{\beta[-\Phi+E-F]}}+\int_{\infty}^{E_{\mathrm{V}}} \mathrm{d} E \frac{G_{\mathrm{e}}(E)}{1+e^{\beta[\Phi-E+F]}} \\
& +N_{\mathrm{D}}\left\{1-\frac{1}{1+e^{\beta[-\Phi+E-F]}}\right\}
\end{aligned}
$$

with $E_{\mathrm{C}}, E_{\mathrm{V}}, F$ being respectively the conduction, valence, and Fermi levels, $G_{e, h}(E)$ the electron (hole) density of states and $N_{\mathrm{D}}$ the dopant density (normalized to $N_{\mathrm{o}}$ ), and $\beta \equiv 1 / \kappa T$. The Fermi level $F$ is obtained from the condition $\rho\left[\left.x_{\mathrm{o}}\right|_{\Phi=0}\right]=0$ at the neutral point $\Phi\left(x_{\mathrm{o}}\right)=0$. This automatically guarantees total charge neutrality over the QW as follows. The point $x_{\mathrm{o}}$ where $\rho\left(x_{\mathrm{o}}\right)=0$ is also the location of the minimum of the screened electric field, since $\mathrm{d} \mathcal{E} /\left.\mathrm{d} x\right|_{x_{0}}=4 \pi \rho\left(x_{0}\right)=0$ there. Now, from $\mathcal{E}(-L / 2)$ $-\mathcal{E}\left(x_{\mathrm{o}}\right)=-\left[\mathcal{E}(L / 2)-\mathcal{E}\left(x_{\mathrm{o}}\right)\right]$ and Gausses law follows $\int_{-L / 2}^{x_{o}} \mathrm{~d} x \rho(x)=-\int_{x_{0}}^{L / 2} \mathrm{~d} x \rho(x)$ and $Q_{-}=-Q_{+}$. The sheath Eqs. 23 and 24 yield the free carrier dielectric shielding inside a plasma-filled QW capacitor of plate charge $\sigma=$ $-\mathcal{E}_{\mathrm{o}} / 4 \pi$ under the nonlinear response $\rho[\Phi]$.

Analytic solutions of (23) and (24) in terms of the polarization field strength $\mathcal{E}$ exist for certain degenerate $e|E-F| \leq \kappa T$ and non-degenerate $e|E-F| \gg \kappa T$ limits. The simplest treatment illustrating all the salient features is the undoped (intrinsic semiconductor) limit $N_{\mathrm{D}}=0$. Since the Fermi level in this case lies close to mid-bandgap and $\left|F-E_{\mathrm{V}, \mathrm{C}}\right| \gg \kappa T$, the non-degenerate Maxwellian limit applies for the carrier statistics. The carrier density is simply given by $N_{e, h}=n_{e, h}^{\mathrm{o}} \exp [\mp e \Phi(x) / \kappa T]$ where $n_{e, h}^{o}=$ $n_{i}=(1 / 4)\left(4 m_{\mathrm{e}}^{*} m_{\mathrm{e}}^{*} \kappa^{2} T^{2} / \pi^{2} \hbar^{4}\right)^{3 / 4} \exp \left[-E_{\mathrm{G}} / 2 \kappa T\right]$ is the zero polarization electron and hole density. Three dimensional density of states is assumed for large enough QW width with small energy spacing $\Delta E_{i} \simeq \kappa T$. Poisson's equation is then simplified to

$\frac{d^{2}}{\mathrm{~d} x^{2}} \Phi=-2 \sin h[\Phi]$.

It has exact analytic solutions, since $x=X(\Phi)$ is given in terms of elliptic integrals of complex argument, and hence $\Phi(x)$ follows in terms of the elliptic amplitude (Jacobi $\left.\operatorname{am}(u)=\sin ^{-1}[\operatorname{sn} u]\right)$ function,

$\Phi\left(x ; V_{\mathrm{L}}, \mathcal{E}_{\mathrm{o}}\right)=\frac{2}{i} \mathrm{am}\left[i(x-L / 2) \sqrt{C}, \frac{2}{C}\right]$

where $V_{\mathrm{L}} \equiv \Phi(L / 2)$ is the potential drop over half the QW length $L$ and $C \equiv 1+\mathcal{E}_{\mathrm{o}}^{2} / 4-\cosh V_{\mathrm{L}}$ (Different profiles apply for given applied voltages [19] across the sheaths.) The field and voltage profiles have respectively even/odd symmetry about the middle of the $\mathrm{QW}, \mathcal{E}(x)=\mathcal{E}(L / 2-$ $x), \Phi(x)=-\Phi(L / 2-x)$, reflecting the opposite electron and hole densities for an undoped material. The opposite polarity electron and hole sheath potentials $V_{e}=-V_{h}=V_{\mathrm{o}}$ are respectively defined by $V_{e} \equiv \Phi(0)-\Phi(L / 2)$ and $V_{h}$ $\equiv \Phi(L / 2)-\Phi(L)$. The corresponding nominal sheath lengths are $L_{e}=L_{h}=L / 2$. However, when $L_{e, h} \gg \lambda_{\mathrm{D}}$, the field in each sheath is essentially localized within a few $\lambda_{\mathrm{D}}$ while the rest of the length is almost field-free.

Solutions and shielded voltage profiles for both Maxwellian, Eq. 26, as well as Fermi-Dirac distributions in general, Eqs. 23, 24, have been given in [15]. Maxwellian profiles are reasonably well fitted with $\sin h$-profiles employed in the present analysis, such as the bottom of the QW Fig. 2a. The screened profiles remain essentially similar for Fermi-Dirac distributions in general, as shown in Fig. 9a, with one difference: the symmetry between the electron and hole charged-layers is broken, $V_{\mathrm{e}} \neq-V_{\mathrm{h}}$. In addition, $\mathrm{F}-\mathrm{D}$ statistics yields higher saturation voltages $V_{\mathrm{S}}$ under given parameters. The saturation values shown in Fig. 7 correspond to general F-D solutions. Finally, for sufficiently small potentials $e V_{\mathrm{o}} / \kappa T \simeq e \mathcal{E}_{\mathrm{o}} \lambda_{\mathrm{D}} / \kappa T \ll 1$ any sheath profiles, including (26), are reduced to exponential profiles [15] $\Phi(x)=V_{\mathrm{o}} \exp (-\sqrt{2} x)$, solutions of the linear differential equation $\frac{d^{2}}{\mathrm{~d} x^{2}} \Phi+2 \Phi=0$.

\section{References}

1. R. Langer, J. Simon, V. Ortiz, N.T. Pelekanos, A. Barski, R. Andr, M. Godlewski, Giant electric fields in unstrained GaN single quantum wells. Appl. Phys. Lett. 74, 3827-3829 (1999)

2. W. Franz, Z. Naturforsch. 13a, 484 (1958).

3. L.V. Keldysh, The effect of a strong electric field on the optical properties of insulating crystals. Soviet Phys. JETP 34, 788-790 (1958)

4. K. Tharmalingam, Optical absorption in the presence of a uniform field. Phys. Rev. 130, 2204-2206 (1963)

5. M. Matsuura, T. Kamizato, Subbands and Excitons in a quantum well in an electric field. Phys. Rev. B 33, 8385-8389 (1986)

6. D.E. Aspnes, Electric-field effects on the dielectric constant of solids. Phys. Rev. 153, 972-982 (1967)

7. B.R. Bennet, R.A. Soref, Electrorefraction and electroabsorption in InP, IGaAs, GaSb, InAs and InSb. IEEE JQE 23, 2159-2166 (1987)

8. D.A.B. Miller, D.S. Chemla, S. Schmitt-Rink, Relation between electroabsorption in bulk semiconductors and quantum wells: The quantum confined Franz-Keldysh effect. Phys. Rev. B 33, 69766982 (1986)

9. H. Shen, F.H. Pollack, Generalized Franz-Keldysh theory of electroabsorption. Phys. Rev. B 42, 7097-7102 (1990)

10. R.J. Elliot, Intensity of optical absoprtion by excitons. Phys. Rev. 108, 1384-1389 (1957)

11. M. Shinada, S. Sugano, Interband optical transitions in extremely anisotropic semiconductors I: bound and unbound exciton transitions. J. Phys. Soc. Jpn. 21, 1936-1946 (1966)

12. D.A.B. Miller, D.S. Chemla, T.C. Damen, A.C. Gossard, W. Wiegmann, T.H. Wood, C.A. Burrus, Electric field dependence of optical absorption near the bandgap of QW structures. Phys. Rev. B 32, 1043-1060 (1986) 
13. S.L. Chuang, S. Schmitt-Rink, D.A.B. Miller, D.S. Chemla. Exciton Green's function approach to optical absorption in a QW with an applied electric field. Phys. Rev. B 43, 1500-1509 (1991)

14. C.Y.P. Chao, S.L. Chuang, Analytical and numerical solutions for a two-dimensional exciton in momentum space. Phys. Rev. B 43, 6530-6543 (1991)

15. S. Riyopoulos, T.D. Moustakas, J.S. Cabalu, Plasma nanosheath formation with carrier accumulation and enhanced localized spontaneous emission at quantum wedges in textured GaN, submitted to Phys. Rev. B 14, 053501 (2007)
16. F. Della Sala, A. Di Carlo, F. Bernardini, V. Fiorentini, R. Scholz, J.-M. Jancu, Free-carrier screening of polarization fields in wurtzite GaN/InGaN laser structures. Appl. Phys. Lett. 74, 2002 (1999)

17. A. Thamm, O. Brandt, J. Ringling, A. Trampert, K.H. Ploog, O. Mayrock, H.-J.Wünsche, F. Hennberger, Optical properties of heavily doped $\mathrm{GaN} /(\mathrm{Al}, \mathrm{Ga}) \mathrm{N}$ multiple $\mathrm{QWs}$ grown on $6 \mathrm{H}-$ $\mathrm{SiC}(0001)$ by reactive molecular-beam epitaxy. Phys. Rev. 61, $16025(2000)$

18. F. Bernardini, V. Fiorentini, Phys. Rev. B 57, R9427 (1998)

19. P. Debye, E. Huckel, Physik. Z 24, 185 (1923) 